Claremont Colleges

Scholarship@ Claremont

Scripps Faculty Publications and Research

Scripps Faculty Scholarship

$1-1-2000$

\title{
Age Differences in the Frontal Lateralization of Verbal and Spatial Working Memory Revealed by PET
}

\author{
Alan Hartley \\ Scripps College \\ Patricia A. Reuter-Lorenz \\ University of Michigan \\ John Jonides \\ University of Michigan \\ Edward E. Smith \\ University of Michigan \\ Andrea Miller \\ University of Michigan \\ See next page for additional authors
}

\section{Recommended Citation}

Reuter-Lorenz, P. A., Jonides, J., Smith, E. E., Hartley, A., Miller, A., Marshuetz, C., \& Koeppe, R. A. (2000). Age differences in the frontal lateralization of verbal and spatial working memory revealed by PET. Journal of Cognitive Neuroscience, 12, 174-187. doi: $10.1162 / 089892900561814$ inclusion in Scripps Faculty Publications and Research by an authorized administrator of Scholarship @ Claremont. For more information, please contact scholarship@cuc.claremont.edu. 
Authors

Alan Hartley, Patricia A. Reuter-Lorenz, John Jonides, Edward E. Smith, Andrea Miller, Christina Marshuetz, and Robert A. Koeppe 


\title{
Age Differences in the Frontal Lateralization of Verbal and Spatial Working Memory Revealed by PET
}

\author{
Patricia A. Reuter-Lorenz, John Jonides, and Edward E. Smith \\ University of Michigan
}

\author{
Alan Hartley \\ Scripps College \\ Andrea Miller, Christina Marshuetz, and Robert A. Koeppe \\ University of Michigan
}

\begin{abstract}
Age-related decline in working memory figures prominently in theories of cognitive aging. However, the effects of aging on the neural substrate of working memory are largely unknown. Positron emission tomography (PET) was used to investigate verbal and spatial short-term storage $(3 \mathrm{sec})$ in older and younger adults. Previous investigations with younger subjects performing these same tasks have revealed asymmetries in the lateral organization of verbal and spatial working memory. Using volume of interest (VOI) analyses that specifically compared activation at sites identified with working memory to their homologous twin in the opposite hemisphere, we show pronounced age differences in this organization, particularly in the frontal lobes: In younger
\end{abstract}

Few domains of human cognition remain untouched by the effects of aging. Such global decline has prompted the search for cognitive mechanisms capable of exerting widespread effects, mechanisms such as working memory, which is known to play a central role in human cognition (Baddeley, 1986; Carpenter \& Just, 1989). Indeed, behavioral research implicates age-related declines in working memory as a source of cognitive aging effects across a variety of domains (for example, Salthouse, 1991). Over the past 10 years, considerable progress has been made toward specifying the neural mechanisms underlying working memory in the human brain (Petrides, Alivisatos, Meyer, \& Evans, 1993; Smith \& Jonides, 1998; cf. Goldman-Rakic, 1992). However, relatively little is known about the effects of normal aging on the circuitry of working memory. Given the central importance of working memory to cognition, and to age-related cognitive decline, we investigated the effects of age on the neural substrate adults, activation is predominantly left lateralized for verbal working memory, and right lateralized for spatial working memory, whereas older adults show a global pattern of anterior bilateral activation for both types of memory. Analyses of frontal subregions indicate that several underlying patterns contribute to global bilaterality in older adults: most notably, bilateral activation in areas associated with rehearsal, and paradoxical laterality in dorsolateral prefrontal sites (DLPFC; greater left activation for spatial and greater right activation for verbal). We consider several mechanisms that could account for these age differences including the possibility that bilateral activation reflects recruitment to compensate for neural decline. of working memory using positron emission tomography (PET).

Working memory refers to the capacity to hold information in mind for short periods of time (that is, 30 sec or less) and to use or manipulate that information in thinking and problem solving tasks. Thus, working memory is thought to include storage mechanisms that maintain its contents in an active state, and executive processes that perform various operations on the stored information (Baddeley, 1986; Jonides, 1995). There is a large body of evidence indicating that the working memory system is composed of verbal and spatial subsystems that are lateralized to the left and right hemispheres, respectively (see Jonides et al., 1996 for a review). This asymmetrical organization was first evident through case reports of the selective loss of verbal working memory following left-hemisphere damage (see Vallar \& Shallice, 1990) and spatial working memory deficits consequent to right-hemisphere damage. The 
lateralized lesions affecting these cases caused dramatic impairments in short-term memory, reducing it's capacity from a normal level of five to seven items to a dysfunctional level of one or two items.

The asymmetrical organization of verbal and spatial working memory has been largely corroborated by neuroimaging studies of healthy, young right-handed adults, particularly for tasks that emphasize short-term information storage (see D'Esposito et al., 1998; Smith, Jonides, \& Koeppe, 1996 for reviews; compare Goldberg, Berman, Randolph, Gold, \& Weinberger, 1996; Owen, Evans, \& Petrides, 1996). Verbal storage tasks have been associated with a predominance of left-hemisphere activation in Broca's area, supplementary motor cortex, premotor cortex, superior and inferior parietal cortex (Awh et al., 1996; Fiez et al., 1996; Jonides et al., 1998; Schumacher et al., 1996). Likewise, during spatial storage tasks, activation predominates in right-hemisphere regions homologous to those active during verbal storage, along with right frontal activation in Brodmann's area (BA 47) and in visual association cortex (that is, BA 18; Jonides et al., 1993). The lateralized frontal components of this circuitry are thought to mediate rehearsal or maintenance of phonological and spatial information represented more posteriorly in the left and right hemispheres, respectively (Awh \& Jonides, 1997; Awh et al., 1996). When executive components are added to the storage tasks, by requiring contextual tagging or manipulation of the stored items, for example, activation of dorsolateral prefrontal sites (DLPFC; BA 45, 46,9) is evident. Differential activation of left and right DLPFC has been reported for such verbal and spatial working memory tasks (e.g., Smith, Jonides, \& Koeppe, 1996), although, overall, the magnitude of asymmetry for these more complex working memory tasks may be less than in simple storage tasks.

How might aging affect the neural circuitry of working memory? A few possibilities present themselves. Prefrontal regions seem particularly vulnerable to the effects of aging (Raz, in press) and changes in frontal lobe function are hypothesized to be the source of various cognitive deficits found in older adults (see Moscovitch \& Winocur, 1995; Raz, in press for reviews). Frontal dysfunction has been linked to age-related impairments in long-term memory, attention and inhibitory processes (Chao \& Knight, 1997; Jonides et al., in press; West, 1996). We might expect then that the anterior components of the working memory system might show greater age-related changes than posterior components. Some recent evidence from a study examining the effects of age on working memory for faces suggests this may the case (Grady et al., 1998).

Another possibility is that aging leads to a decline in the functional specificity of the working memory system. Neuroimaging evidence for declining specificity comes from a PET study of the "what" vs. "where" processing streams in older and younger adults (Grady et al., 1992, 1994). Subjects performed a face-matching task, intended to activate the what/ventral stream, or a location-matching task designed to activate the where/ dorsal stream. Younger participants showed the expected dissociation between dorsal and ventral stream processing: face-matching produced more ventral stream activation and location-matching evoked more dorsal stream activation. In contrast, older adults showed significant activation of dorsal and ventral sites during both tasks. More recently, studies of episodic memory have demonstrated that older adults depart from the pattern of lateralized prefrontal activity that has been shown to differentiate encoding and retrieval processes in younger adults (that is, Cabeza et al., 1997; Grady et al., 1995; Madden et al., 1999; Tulving, Kapur, Craik, Moscovitch, \& Houle, 1994). Some studies report more bilateral activation in older than younger adults during retrieval, and others find greater left than right activation in anterior sites where younger subjects show the opposite pattern.

We might expect then that aging would also alter the lateral organization of the working memory system. Here, we report PET evidence of age differences in the asymmetrical organization of verbal and spatial working memory particularly in the frontal lobes: for younger adults, activation is predominantly left lateralized for verbal working memory, and right lateralized for spatial working memory, whereas older adults show activation in left and right frontal sites for both types of memory.

We tested two groups of older (62-75 years) and younger (18-30 years) female ${ }^{1}$ adults in two working memory tasks that emphasized short-term information storage and had the same basic structure (Figure 1). In the verbal-storage task, subjects were presented with four target letters, they stored them in memory for $3 \mathrm{sec}$, and then they indicated whether or not a probe letter matched any target. In the spatial-storage task, three target locations were marked on the screen, subjects stored them for $3 \mathrm{sec}$ and then indicated whether or not a probe location matched any target location. Each memory task was paired with a control task designed to include all of the cognitive components of the memory task (for example, perceptual encoding, response selection, preparation, and initiation) except the working memory component (that is, immediate matching). Thus, subtracting the images of the control condition from the images of the memory condition should reveal primarily the brain areas that are active during verbal or spatial working memory, respectively. It is important to bear in mind that because our PET analyses rely on subtraction logic, the results reflect activation changes in the memory task relative to the control tasks for each age group, and not age differences in the absolute levels of neural activity associated with working memory processes. Thus, any age differences we report could be due to group differences in the 


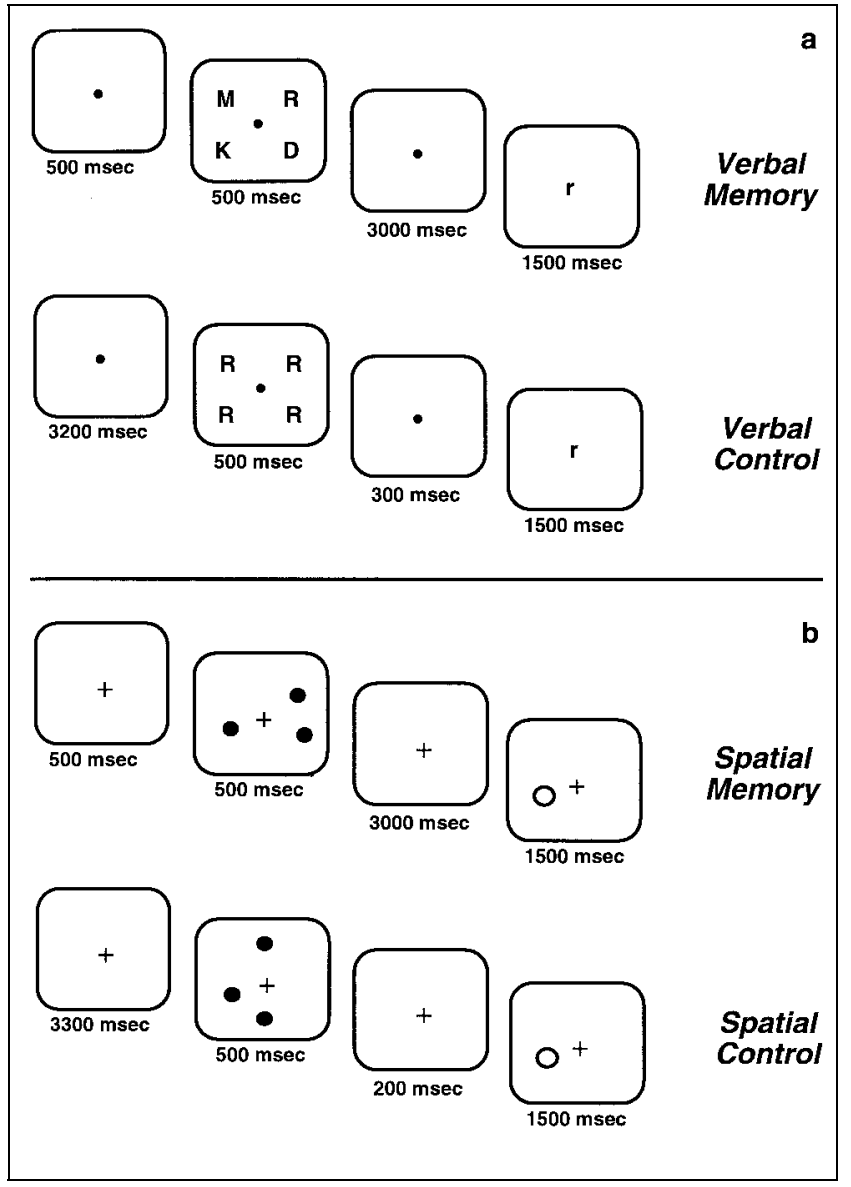

Figure 1. Cognitive tasks. Sequence of events and timing parameters for the verbal (a) and spatial (b) working memory and control tasks. For the spatial control task, only one dot location varies across trials; the top and bottom dots appear in the same locations for each trial.

control conditions or the memory tasks. Although we have no way to know for certain, we assume that age has a greater effect on the neural substrate of working memory than on the substrate of the immediate matching tasks, and that this age effect is evident in our differences images.

\section{RESULTS}

\section{Verbal Working Memory}

\section{Performance Data}

Both groups showed nearly perfect accuracy on the control task, scoring 99\% correct or higher; however, an analysis of variance indicated that the younger subjects were faster than the elderly on both tasks (control task: $F(1,22)=6.17 ; p=.02$; memory task: $F(1,22)=29.9 ; p=.0001)$ and more accurate on the memory task $(F(1,22)=6.14 ; p=.02$; see Table 1$)$.

\section{PET Data}

The averaged subtraction images indicate approximately $1.0 \%$ more activation in the younger adults than the older group (see Table 2). Due to the relatively small sample size, however, only a site in left thalamus and right cerebellum reached conventional levels of significance using post hoc tests in the younger group. In order to establish that the present results are nonetheless representative of the verbal working memory circuitry in the younger brain, regions of interest (ROIs) derived from our previous results using this task (see Awh et al., 1996; Smith et al., 1996; Experiment 1) were applied to the current data set from the young subjects. Seven of the nine regions were significant at the $p<.05$ level (regions in Broca's area, BA 6 , BA $40 / 7$ on the left, and anterior cingulate). The remaining two were significant at the .10 level (left insular, right cerebellum). Likewise, when the nine most active regions from the present study were applied to the data set reported by Smith et al.

Table 1. Mean Accuracy (Proportion Correct), Response Times and Standard Errors for Younger and Older Participants in the Verbal and Spatial Working Memory Tasks with Each Corresponding Control Condition

\begin{tabular}{|c|c|c|c|c|c|}
\hline & & & & & \\
\hline & & Accuracy & Response Time & Accuracy & Response Time \\
\hline Younger & Control & 0.99 & 524 & 0.99 & 529 \\
\hline & & 0.003 & 28 & 0.195 & 23 \\
\hline & Memory & 0.97 & 654 & 0.91 & 706 \\
\hline & & 0.017 & 28 & 1.41 & 17 \\
\hline Older & Control & 0.99 & 610.9 & 0.99 & 741 \\
\hline & & 0.004 & 20 & 0.28 & 23 \\
\hline & Memory & 0.92 & 843 & 0.92 & 915 \\
\hline & & 0.012 & 20 & 1.38 & 22 \\
\hline
\end{tabular}


(1996), all but one was significant at the $p<.05$ level (left BA 7). This reciprocity establishes that the results obtained with this working memory task are replicable in younger adults.

Post hoc analyses of the activation images from the older adults showed significant activation in area 6 bilaterally, left cerebellum and right dorsolateral prefrontal area 46/9 (see Table 2). Thus, post hoc analyses suggest laterality differences between the two age groups. In order, specifically, to test age differences in laterality, we devised an analysis that targeted the verbal working memory circuitry as delineated by previous published reports. Although these studies have all used younger adults as participants and, therefore, may not be fully representative of the circuitry of older adults, we nevertheless constructed ROIs from these previous studies because they are the basis of our neuroanatomical knowledge about working memory. To minimize the influence of any idiosyncratic effects in any one study, we culled ROIs from a range of PET studies (Awh et al., 1996; Fiez et al, 1996; Grasby et al., 1993; Paulesu, Frith, \& Frackowiak, 1993; Petrides et al., 1993; Salmon et al., 1996; Schumacher et al., 1996). Figure 2a illustrates the locations of these ROIs in a sagittal projection that collapses across the $x$ plane in Talairach space. We assessed the laterality of activations within each age group by comparing activation in each region of interest with the activation in the homologous site in the opposite hemisphere.

\section{Laterality Comparisons in PET}

A set of 85 ROIs was derived from published reports of peak pixels active during PET studies of verbal working memory tasks, including our own (see Figure 2). The majority of these regions were in the left hemisphere and include frontal sites in BA 45, 46, 10, and 9, 44 (Broca's area), 6 (premotor and supplementary motor sites), parietal sites in areas 40 and 7 , and temporal sites in 42 and 22. Spherical volumes $1.5 \mathrm{~cm}$ in diameter (volumes of interest, VOIs) were constructed around reported peak pixels, replicated in the symmetrical location in the opposite hemisphere, and then applied to each subject's memory-minus-control subtraction images, thereby permitting a comparison of the average activation changes across homologous regions. To assess differences in asymmetry in the front and back of the cortex, an anterior subset $(n=34)$ was defined in Talairach space as those ROIs with a $y>0$ and a posterior subset ( $n=33$ ) with $y<-10$ and $z>-10$.

The results from paired $t$ tests comparing average activation for each specified subset of VOIs against zero are illustrated in Figure $3 \mathrm{a}$ and $3 \mathrm{~b}$. The first thing to notice about Figure 3 is that older and younger adults show approximately the same activation across the entire set of VOIs, and, in some cases, older adults show greater activation than the younger group. These features of the data argue that this VOI analysis was not systematically biased against finding activation in the older group. For the posterior VOIs, both groups showed significant activation in the left hemisphere (paired $t$ tests evaluated vs. 0: older: $t(15)=5.96$, $p=.0001$; younger: $t(7)=3.971, p<.003)$ and neither group showed any hint of reliable activation in the set of right-hemisphere VOIs (older: $t(15)=.04, p=.45$; younger: $t(7)=-.112, p=.46$; see Figure $2 \mathrm{~b}$ ). For both groups, this left/right activation difference was reliable (older: $t(15)=3.795, p=.0009$; younger: $t(7)=4.793$, $p=.001)$. Moreover, there was no difference between the groups in the magnitude of this asymmetry (unpaired $t$ test comparing average left-right differences for younger vs. older groups (that is, difference of differences, $t(22)=.014, p=.495$; all tests one-tailed).

A strikingly different pattern is evident for the anterior VOIs (see Figure 3a). Again, the younger adults show asymmetrical activation: There is significant net activation in the left VOIs $(t(7)=4.96$, $p=.0008)$ but not the right $(t(7)=.993, p=.178)$, and this left-right activation difference approaches significance $(t(7)=1.76, p=.061)$. In contrast, for older adults, the anterior VOIs in both hemispheres are significantly active (left: $t(15)=3.36, p=.002$; right: $t(15)=4.60, p=.0002)$ and there is no difference in the degree of left-hemisphere and right-hemisphere activation $(t(15)=-1.15, p=.134)$. Indeed, the magnitude of asymmetry is significantly different between the two age groups (unpaired $t$ test comparing average left-right differences for younger vs. older groups: $t(22)=2.15, p=.021$ ) .

This anterior subset of VOIs was further divided into four major subregions (medial SMA, lateral SMA, DLPFC (46/9), Broca's area (44)), and age differences in laterality were examined in these areas separately. Several anterior VOIs $(n=7)$ that were part of the larger subset were not included in this analysis because they were anatomically disparate and there were too few from any one region (that is, three or less) to constitute a separate subregion. At this finer grain, the results are more complex and less robust, however, there are several noteworthy patterns. As can be seen from Table 3, both groups showed bilateral activation in the VOIs within medial SMA, which may have resulted in part from the size of the VOIs and from the limited spatial resolution of PET. In lateral SMA, both groups tended toward greater left than right activation, with the older adults showing stronger asymmetry than the younger group. Broca's area was highly activated on the left and deactivated on the right in the younger group, whereas older adults showed significant activation bilaterally. In DLPFC, the younger group showed significant activation only on the left whereas the older group showed significant activation on the right with only a weak tendency toward left activation in this region. Thus, in contrast with the left lateralized activity in the younger 
Table 2. Regions of Significant Task-Related Activity for Both Age Groups in the Verbal and Spatial Tasks

\begin{tabular}{|c|c|c|c|c|c|}
\hline \multirow[b]{2}{*}{ Brodmann's Area } & \multicolumn{3}{|c|}{ Stereotactic Coordinate } & \multirow[b]{2}{*}{ Percent Change } & \multirow[b]{2}{*}{ z Score } \\
\hline & $x$ & $y$ & $z$ & & \\
\hline \multicolumn{6}{|l|}{ Verbal } \\
\hline \multicolumn{6}{|c|}{ Younger subjects $(n=8)$} \\
\hline Left thalamus & 6 & -17 & 2 & 5.32 & 4.54 \\
\hline Right cerebellum & -33 & -53 & -25 & 5.02 & 4.29 \\
\hline Right 6 & -3 & 1 & 52 & 4.94 & 4.22 \\
\hline Left 6 & 8 & 8 & 47 & 4.81 & 4.10 \\
\hline Left 6 & 48 & -1 & 29 & 4.80 & 4.09 \\
\hline Left 32 & 1 & 12 & 38 & 4.78 & 4.08 \\
\hline Left 40 & 33 & -51 & 38 & 4.64 & 3.96 \\
\hline Left 4 & 30 & -8 & 45 & 4.61 & 3.93 \\
\hline Left 7 & -30 & -58 & 43 & 4.49 & 3.83 \\
\hline \multicolumn{6}{|c|}{ Older subjects $(n=16)$} \\
\hline Right $6 / 32$ & -1 & 8 & 45 & 4.63 & 5.76 \\
\hline Left $6 / 4$ & 48 & -1 & 22 & 4.63 & 5.76 \\
\hline Left cerebellum & 15 & -64 & -16 & 4.49 & 5.58 \\
\hline Right $46 / 9$ & -39 & 35 & 29 & 4.17 & 5.19 \\
\hline Left 6 & 30 & 3 & 47 & 3.46 & 4.30 \\
\hline Right cerebellum & -28 & -53 & -22 & 3.39 & 4.21 \\
\hline Left 17 & 8 & -87 & 9 & 3.27 & 4.06 \\
\hline Left $7 / 40$ & 35 & -58 & 43 & 3.27 & 4.06 \\
\hline Left 40 & 44 & 21 & 20 & 3.25 & 4.03 \\
\hline \multicolumn{6}{|l|}{ Spatial } \\
\hline \multicolumn{6}{|c|}{ Younger subjects $(n=10)$} \\
\hline Left $7 / 19$ & 24 & -64 & 40 & 2.98 & 5.34 \\
\hline Left 40 & 37 & -49 & 38 & 2.86 & 5.14 \\
\hline Right 40 & -42 & -40 & 38 & 2.79 & 5.01 \\
\hline Left 6 & 24 & -8 & 47 & 2.72 & 4.89 \\
\hline Right 7 & -17 & -62 & 52 & 2.53 & 4.54 \\
\hline Right 6 & -24 & -1 & 54 & 2.25 & 4.04 \\
\hline \multicolumn{6}{|c|}{ Older subjects $(n=10)$} \\
\hline Left $4 / 6$ & 28 & -8 & 50 & 2.89 & 5.93 \\
\hline Right 7 & -12 & -58 & 47 & 2.82 & 5.79 \\
\hline Left 7 & 19 & -67 & 45 & 2.67 & 5.47 \\
\hline Right 40 & -33 & -53 & 40 & 2.57 & 5.27 \\
\hline Right 19 & -24 & -64 & 34 & 2.56 & 5.26 \\
\hline Left 40 & 46 & -49 & 40 & 2.39 & 4.91 \\
\hline
\end{tabular}


Table 2. (continued)

\begin{tabular}{lccccc}
\hline & \multicolumn{3}{c}{ Stereotactic Coordinate } & & \\
\cline { 2 - 4 } Brodmann's Area & $x$ & $y$ & $z$ & Percent Change & $z$ Score \\
\hline Spatial & & & & & \\
Left 7 & 30 & -58 & 47 & 2.28 & 4.67 \\
Right 19 & -30 & -73 & 20 & 2.16 & 4.44 \\
Right cerebellum & -30 & -55 & -22 & 2.12 & 4.36 \\
Right 6 & -46 & 8 & 32 & & 4.36 \\
\hline
\end{tabular}

The thresholds for significant $z$ scores with correction for multiple comparisons are as follows: Verbal: younger $=4.23$, older $=4.35$; spatial: younger $=4.23$, older $=4.35$. Note that for the verbal task, the presence of fewer significant sites for the younger subjects is due to the smaller $n$, not smaller magnitude changes. In addition, note that the magnitudes of the activations for the spatial task are lower than for the verbal task. This is in part, but not entirely, due to the increased scatter fraction in 3-D (spatial study) compared to 2-D (verbal study) scans, which causes up to a $20 \%$ reduction in the apparent activation magnitude. However, reduced statistical noise in the 3-D study more than compensates, and, thus, the $z$ score (signal-to-noise ratio) for a given magnitude change is higher for the spatial study.

group, the global picture of bilateral anterior activation in the older adults is the result of several underlying, but weaker patterns: activation of left lateral SMA, bilateral activation of Broca's area, and activation of right DLPFC. The patterns of activation in these frontal subregions reveal that the global anterior bilaterality in older adults is a composite picture of a verbal working memory circuitry whose components are distributed across both hemispheres. We note that the bilateral activation of Broca's area and right DLPFC activation that are unique to the older group has been replicated in a different data set from our lab (Jonides et al., in press) and will be the focus of a separate report. We consider the functional significance of these patterns after presenting the results from the spatial working memory task.

\section{PET-Performance Relations}

In order to evaluate whether the patterns of laterality observed in our two age groups are related to task performance, we conducted a series of correlational analyses. For the PET data, we derived difference scores by subtracting the net activation levels in the right VOIs from the net activation in the left VOIs reported above. We computed three difference scores for each subject for the anterior and posterior subsets, and for the entire set of VOIs. In addition to using the difference scores, correlations were also performed using the net activations for the left and right anterior, posterior, and fullhemisphere VOIs. The percent correct score and average reaction time for the working memory task performed during PET were transformed into $z$ scores based on separate distributions for each group. In addition, for each subject we calculated a combined $z$ score by subtracting the $z$ score for reaction time from the $z$ score for accuracy (subtraction was used because better performance is indicated by higher accuracy but by lower response time, and so subtraction adjusts for this reciprocal relationship; Salthouse, 1992). These correlations were computed for the younger and older subjects separately and for the two groups combined.

Only two correlations reached significance and these were for the older group. The combined $z$ score was positively correlated with the magnitude of activation for all the left ROIs combined, and for the left posterior subset alone $(p<.05$, uncorrected for multiple correlations). This significant correlation indicates that greater left-hemisphere activation, particularly in posterior regions, is associated with better performance in the older group. The correlation between the combined $z$ and left anterior subset was not significant for the older group, nor were any of the correlations for the younger or combined groups. Finally, the three performance measures were also correlated with the left and right activation levels for each of the four anterior subregions, and the left-right difference score for each region. Only two correlations reached significance at a level of $p<.01$ : For the older group, activation of left Broca's area was negatively correlated with response time, and for the younger group, the activation of left lateral SMA was negatively correlated with response time.

In a further attempt to relate activation patterns to performance, we undertook an additional analysis. A median split was used to group the older adults based on their response speed because this measure spanned a wider range of performance than did accuracy. The laterality of DLPFC activation differed significantly ( $t$ test comparing left-right difference scores for the two subgroups: $t(14)=2.50, p<.013)$. The fast group showed DLPFC activation bilaterally, whereas for the slow group, only right DLPFC was activated, coupled with nonsignificant deactivation of left DLPFC. Both groups showed bilateral activation of Broca's area.

\section{Spatial Working Memory}

The first experiment provides clear evidence that the pattern of laterality associated with the anterior compo- 


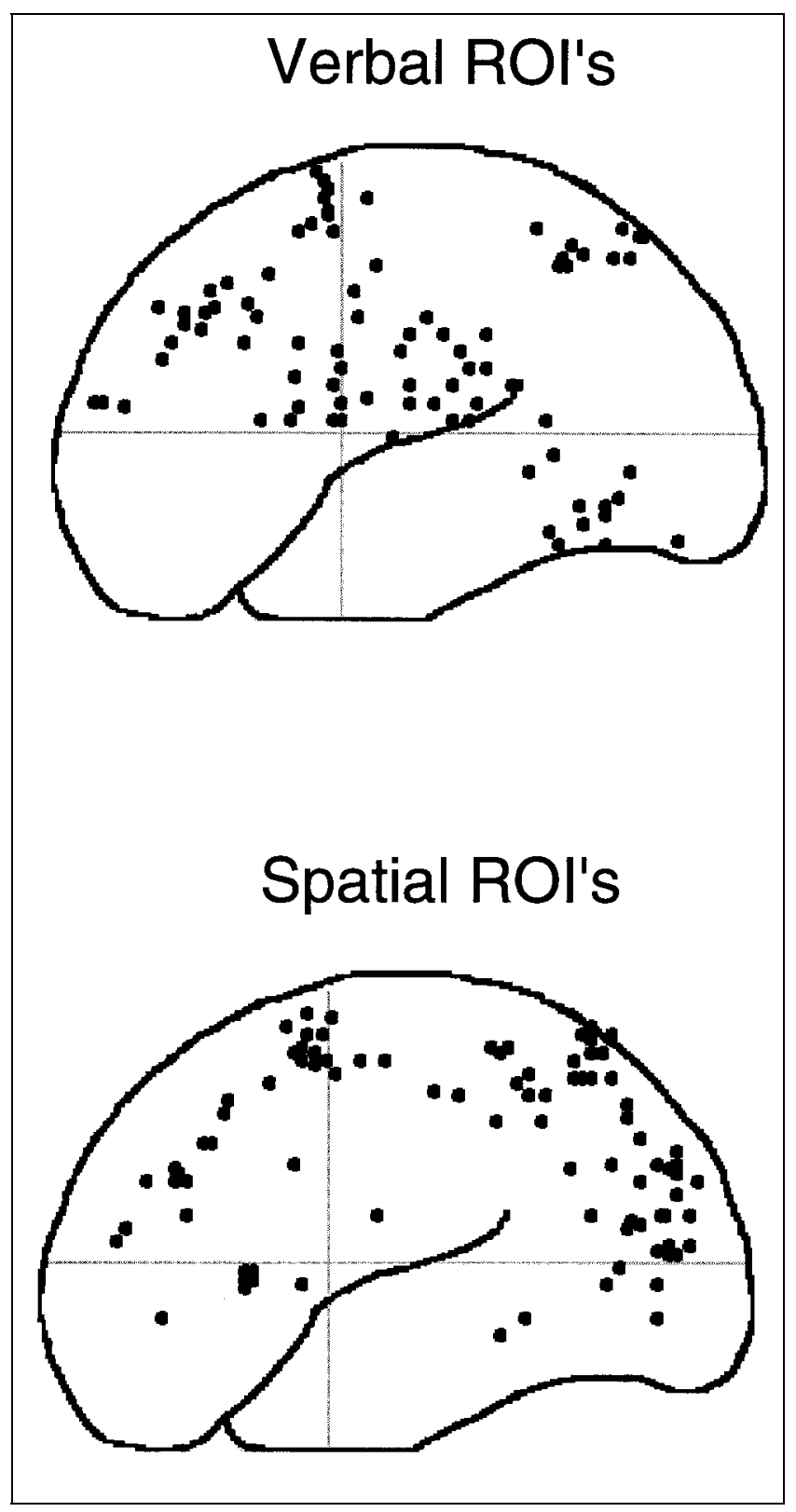

Figure 2. These figures indicate the location of the verbal (top) and spatial (bottom) regions of interest obtained from prior studies that used PET to study verbal and spatial working memory. Because these glass brain images collapse across the $x$-axis (left/right) they reveal only the $y$ (anterior/posterior) and $z$ (dorsal/ventral) of location each volume. To compare the laterality of the working memory circuitry in older and younger adults, volumes were formed around each of these regions and a twin volume was created in the homologous site in the opposite hemisphere (see text for details). Laterality was assessed by comparing the activity in each volume with the activity in its homologous twin.

nents of the verbal working memory system differs in older and younger adults. Given that verbal and spatial working memory are characterized by opposite patterns of asymmetry in the younger brain, we might expect that the circuitry of spatial working memory, like verbal working memory, would show age differences in laterality. Indeed, the following experiment confirmed this prediction.
Two new groups of younger and older adults participated in the spatial memory and control tasks illustrated in Figure $1 \mathrm{~b}$ while PET images were obtained. The older adults who participated in this experiment met an accuracy criterion (see Methods section below). This was done to maximize the similarity in performance of younger and older participants, and to insure that any age-related accuracy differences on this task did not significantly exceed those that were present in the verbal task.

\section{Performance Data}

There were no group differences in control or memory accuracy, although the responses of senior subjects were significantly slower (control: $F(1,18)=18.22, p=.0005$; memory: $F(1,18)=21.1, p=.0002$; see Table 1$)$.

\section{PET Data}

The averaged subtraction images revealed approximately equivalent levels of activation in the two age groups (see Table 2). Post hoc analyses revealed peaks of significant activation in parietal areas 7 and 40 in both hemispheres and in premotor cortex bilaterally in younger and older subjects. It is apparent from Table 2 that the activations from the spatial task are lower in magnitude than in the verbal task for both groups. Nevertheless, the significance levels of the spatial memory activations are high because there are more scans per condition, and the scanning was done in 3-D thereby improving the signal-to-noise ratio (see Methods section). In addition, the present results replicate previous results from this lab (Jonides et al., 1993) and vice versa; the four ROIs from this prior work were also active in our present sample of younger subjects (all $p$ 's $<.05$; right BA 40, 6, 47, 19). Likewise, the six most active sites from our present young sample were significantly activated in the original subject group.

\section{Laterality Comparisons in PET}

Age differences in laterality were evaluated as in the verbal study, using a new set of 98 regions from published reports of spatial working memory tasks (Courtney, Ungerleider, Keil, \& Haxby, 1996; Goldberg et al., 1996; Owen et al., 1996; Smith et al, 1996, 1995; see Figure 2). The majority of these regions were in the right hemisphere. The anterior subset $(n=32 ; y>0)$ includes frontal sites in BA 9, 46, 47, premotor and supplementary motor cortex. The posterior sites $(n=59 ; y<-10$ and $z>-10$ ) include parietal (BA 40, 7), striate, and extrastriate loci (BA 18, 19) and precuneus (BA 31). For the posterior set, both groups showed significant net activation in the left and right hemispheres (older: left $t(9)=2.46, p<.018$; right $t(9)=4.27, p=.001$; younger: 


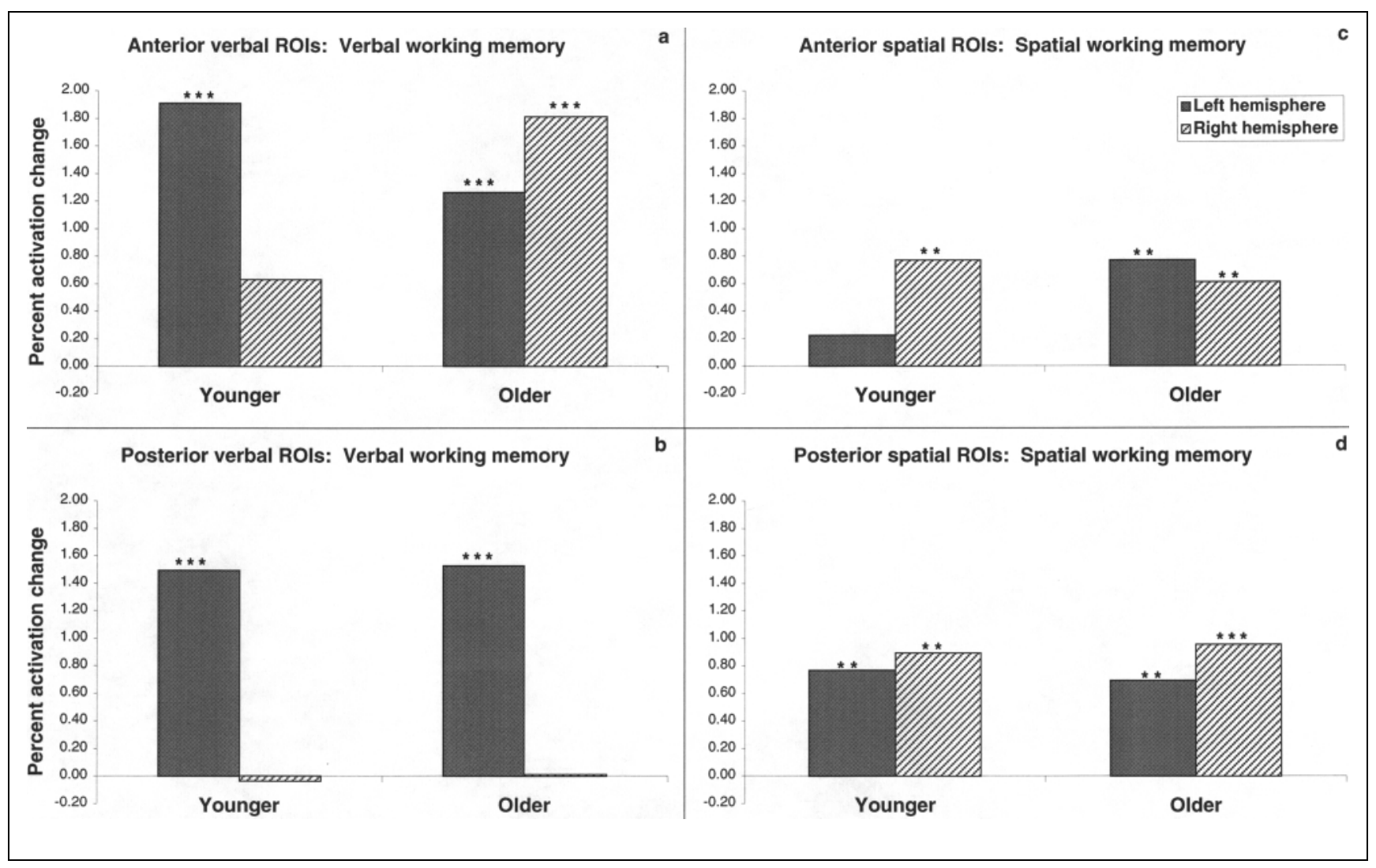

Figure 3. Working memory induced activation changes. Graphs depict percent change in activation for the memory minus control subtractions for the verbal and spatial tasks. Asterisks denote significance levels when comparing activation changes to zero using one-tailed tests: ${ }^{*} p<.10 ; * * p<.03$; $* * * p<.001$. The graphs demonstrate the lack of age differences in the posterior VOIs for the verbal (b) and spatial (d)tasks. In contrast, the anterior VOIs demonstrate lateralized activations for younger adults, but bilateral activations for older adults (verbal (a) and spatial (b)).

left $t(9)=2.81, p=.010$; right $t(9)=2.32, p=.022$; see Figure 3d). For both groups, the magnitude of posterior left-hemisphere and right-hemisphere activation was approximately equivalent (older: left vs. right $t(9)=.851, p=.208$; younger: left vs. right $t(9)=1.11$, $p=.292$ ). An unpaired $t$ test comparing the average right-left differences for younger vs. older groups was not significant: $t(18)=.356, p=.363$. Finding posterior bilaterality in both subject groups for this spatial task is consistent with other cognitive neuroscience evidence for left-hemisphere and right-hemisphere contributions to visuospatial processes (Ivry \& Robertson, 1998).

The pattern is markedly different for the anterior VOIs (see Figure 3c). Younger adults show significant activation only in the right hemisphere (left $t(9)=.532$, $p=.304$; right $t(9)=2.21, p<.027)$, whereas older adults show significant activation in both hemispheres (left $t(9)=2.31, p=.022$; right $t(9)=2.14, p=.030)$. As in the verbal data set, the younger group shows significantly lateralized activity, and the older group does not (younger: right vs. left, $t(9)=-1.82, p=.050$; older: right vs. left, $t(9)=.415, p=.343)$. This age difference in activation asymmetry approaches significance (unpaired $t$ test comparing right-left difference scores for younger vs. older groups: $t(18)=-1.54, p=.070$ ).
Within the anterior subset of VOIs, three main subregions were identified and examined separately for differences in laterality: lateral SMA, DLPFC (BA 46/9), ventrolateral prefrontal cortex (VPFC, BA 47). Nine VOIs from the anterior subset were not included in this analysis because they did not form an anatomically cohesive subregion with more than three VOIs. For the younger group, activation in each of the three subregions was right lateralized, with $p$ values differing from zero at the $p<.05$ level for right SMA, and right VPFC, along with a nonsignificant tendency toward greater right than left DLPFC activation (see Table 3). Tested against zero, the older group showed activation in left and right SMA, left DLPFC, and right VPFC (all $p$ 's <.05). Note that in the post hoc analysis reported in Table 2, both groups show peaks of activation in left and right lateral premotor cortex. The VOI analysis probed regions of SMA that were anterior to left-sided peaks that emerged in the post hoc analyses $(y>1$ and $y=-8$, respectively). Taken together, the post hoc and VOI analyses reveal that the older group had significant SMA/premotor activity bilaterally, whereas in the younger group, left activation was restricted to the posterior premotor region. Thus, as with the verbal data, the components of the 
Table 3. Mean Percent Activation Change in the Anterior Subregions of the Verbal and Spatial VOIs

\begin{tabular}{|c|c|c|c|c|c|c|c|c|}
\hline \multirow[b]{2}{*}{ Verbal } & & \multicolumn{3}{|c|}{ Younger Adults } & \multicolumn{3}{|c|}{ Older Adults } & \multirow{2}{*}{$\begin{array}{c}\text { Young vs. Old } \\
L-R\end{array}$} \\
\hline & & Left & Right & $L-R$ & Left & Right & $L-R$ & \\
\hline \multirow[t]{2}{*}{ Broca's } & $\%$ change & 2.61 & -1.06 & 3.61 & 2.66 & 0.88 & 1.77 & \\
\hline & $p$ value & 0.0015 & 0.203 & 0.0028 & 0.0003 & 0.02 & 0.19 & 0.077 \\
\hline \multirow[t]{2}{*}{ SMA lat. } & $\%$ change & 0.75 & -0.002 & 0.75 & 1.39 & 0.28 & 1.12 & \\
\hline & $p$ value & 0.21 & 0.49 & 0.20 & 0.008 & 0.31 & 0.06 & 0.378 \\
\hline \multirow[t]{2}{*}{ SMA med. } & $\%$ change & 3.40 & 3.16 & 0.28 & 3.10 & 3.30 & -0.20 & \\
\hline & $p$ value & 0.01 & 0.019 & 0.19 & 0.0002 & 0.0002 & 0.306 & 0.212 \\
\hline \multirow[t]{2}{*}{ DLPFC } & $\%$ change & 2.03 & 0.77 & 1.26 & 0.60 & 2.53 & -1.93 & \\
\hline & $p$ value & 0.006 & 0.267 & 0.20 & 0.17 & 0.0004 & 0.009 & 0.019 \\
\hline \multicolumn{2}{|l|}{ Spatial } & Left & Right & $R-L$ & Left & Right & $R-L$ & $R-L$ \\
\hline \multirow[t]{2}{*}{ SMA lat. } & $\%$ change & 0.61 & 1.17 & 0.56 & 1.29 & 0.85 & -0.62 & \\
\hline & $p$ value & 0.14 & 0.014 & 0.198 & 0.047 & 0.046 & 0.322 & 0.19 \\
\hline \multirow[t]{2}{*}{ VPFC } & $\%$ change & -0.17 & 0.60 & 0.77 & 0.25 & 0.77 & 0.52 & \\
\hline & $p$ value & 0.33 & 0.03 & 0.09 & 0.28 & 0.015 & 0.22 & 0.34 \\
\hline \multirow[t]{2}{*}{ DLPFC } & $\%$ change & -0.15 & 0.62 & 0.77 & 0.71 & 0.12 & -0.59 & \\
\hline & $p$ value & 0.38 & 0.19 & 0.08 & 0.047 & 0.37 & 0.066 & 0.02 \\
\hline
\end{tabular}

$T$-tests comparing each region to zero were liberal in that they were not corrected for multiple comparisons, and they are one-tailed. The $p$ values for these tests are reported beneath the mean percentage of activation change for each region, except in the last column, which reports the $p$ values for unpaired tests comparing the left-right differences scores from the younger and older groups.

anterior working memory circuitry tend to be distributed across the left-hemisphere and right-hemisphere in older adults, whereas for the younger group this circuitry is more lateralized.

\section{PET-Performance Relations}

For the spatial working memory performance and PET, we performed the same sets of correlations described above for the verbal task. None of these correlations reached significance. A median split of fast and slow performers also yielded no significant differences, most likely due to the small number of subjects in each group, and the lack of variability in response speed.

\section{GENERAL DISCUSSION}

The results from this investigation show pronounced age differences in activation of the anterior components of the neural circuitry associated with verbal and spatial working memory. Younger adults show opposite patterns of lateralized frontal activity depending on the type of material being retained in working memory, with greater left-hemisphere activation for verbal materials and greater right-hemisphere activation for spatial materials. By contrast, older adults engage left and right frontal regions for both types of memory. Analyses of separate subregions of frontal cortex generally corroborate the opposite patterns of laterality for verbal and spatial memory in younger adults. These subregion analyses also reveal that the overall picture of bilateral anterior activation in older adults results from several underlying patterns. In contrast to the younger group, older adults showed bilateral activity in BA 44 (Broca's area) and lateral SMA in the verbal and spatial tasks, respectively. In addition, they showed a paradoxical pattern of lateralization in DLPFC, with greater right than left activation in this region in the verbal task, and greater left than right activation in the spatial task. It should be noted, however, that some of the patterns of activation within the anterior subregions are less robust than the global picture of anterior bilaterality in the older adults. Nonetheless, we offer some speculations about the functions of these component patterns that are amenable to further research.

In the present verbal memory results, as well as several others from this laboratory, younger adults show activation in Broca's area coupled with nonsignificant deactivation in the homologous site in the opposite hemisphere (for example, Jonides et al., in press). In contrast, older adults in the present study showed significant activation in Broca's area and in its right-hemisphere homologue. In addition to its putative role in speech production and other linguistic processes, Broca's area is considered to be part of the verbal working memory rehearsal circuit that maintains phonological information in an active state over a short retention interval (for example, Awh et al., 1996). It has been hypothesized that inhibitory interactions between Broca's area and its right-hemisphere homologue normally limit right-sided contributions to lan- 
guage processing and that the latent linguistic abilities of the right hemisphere can facilitate recovery from aphasia (Kinsbourne, 1971). Indeed, several neuroimaging studies of patients with language recovery or compensation after focal left-hemisphere damage (that included Broca's area in some cases) report activation in the right frontal operculum (BA 44/45) which indicates a capacity for this region to participate in language processes (Buckner, Corbetta, Schatz, Raichle, \& Petersen, 1996; Weiller et al., 1995). Other reports of recovery from deficits indicate that bilateral activation of homologous regions could be a general mechanism of compensatory processing (for example, Engelien et al., 1995). In view of these results, it seems feasible that the bilateral activation of Broca's area in the older adult group reflects compensatory recruitment that maintains the functioning of the rehearsal circuit in the aging brain.

In spatial working memory, lateral regions of SMA have been attributed a prominent role in rehearsal processes that maintain location information in an activated state (Awh \& Jonides, 1997; Courtney, Petit, Maisog, Ungerleider, \& Haxby, 1998). VOIs within this frontal subregion were uniquely activated in both hemispheres in the older group in our spatial working memory task, whereas in the younger group this subregion was activated primarily on the right. The evidence for greater bilaterality in this area for older than younger subjects is certainly less robust than the results for Broca's area in the verbal task. Thus, while we cautiously suggest that compensatory recruitment also characterizes the pattern of activation associated with visuospatial rehearsal, this interpretation must be regarded as speculative.

The other subregion that contributes to the global pattern of age differences in anterior laterality is DLPFC. In both tasks, older adults show a laterality pattern that is opposite to that shown by the younger group. In general, activation of DLPFC is characteristic of working memory tasks that require information storage, plus processing and manipulation of the stored contents (for example, D'Esposito et al., 1998; Smith \& Jonides, 1999). In studies of human working memory, DLPFC has, thus, been identified with executive processes rather than rehearsal. Such DLPFC activation tends to be significant in both hemispheres (for example, Jonides et al., 1997), however, when asymmetries are observed, they occur in the expected direction with left-hemisphere predominance for verbal materials and right-hemisphere predominance for spatial materials (see D'Esposito et al., 1998; Smith et al., 1996). One recent exception may shed light on the present results. Rypma, Prabhakaran, Desmond, Glover, \& Gabrieli (1999) found bilateral DLPFC activation in younger adults, greater on the right than left, in a verbal-storage task but only for a memory load of six items. Smaller memory loads activated predominantly left-hemisphere sites, and did not activate DLPFC in either hemisphere. Rypma et al. suggest DLPFC activation reflects the recruitment of executive processes to augment simple rehearsal processes that may be inadequate for retaining larger memory loads. According to this argument, the right DLPFC activation in older adults reflects their need to invoke executive processes at lighter memory loads compared to younger adults. Likewise, left DLPFC activation in the spatial task could augment rehearsal processes that are otherwise inadequate to meet the memory demands. Unfortunately, this interpretation of the present results for older adults does not clarify why the laterality of the DLPFC activation is opposite to the pattern typically found for verbal and spatial tasks.

Age differences in DLPFC activation and its laterality have been reported in several other studies with different stimulus materials and different task demands. Grady et al. (1998), for example, found left DLPFC activation that was unique to older adults in a working memory task using faces. Cabeza et al. (1997, in press) found age differences in prefrontal activation in BA 10 during an episodic retrieval task (see also Madden et al., 1999). There have been numerous speculations about the function of these age differences, including age-related increases in attentional processing or semantic/associative processing (see Grady et al., 1998 for a review). It is unclear how any of these suggestions would account for the paradoxical patterns of DLPFC activation that we observed for verbal and spatial working memory. Nevertheless, it seems plausible that the present effects reflect age differences in the recruitment of executive processes mediated by DLPFC. The question of whether age alters executive processing brought to bear on encoding, maintenance, or retrieval from short-term memory can ultimately be addressed with the temporal resolution of functional MRI in future studies. Whatever its role, there is some suggestion from the present data set that paradoxical laterality is less efficient. In the verbal task, slower adults showed greater right than left DLPFC activation, whereas the faster group showed bilateral DLPFC activation.

The age-related differences that we have observed naturally raise the question of whether older and younger adults are using different strategies, or whether the same cognitive strategies in the older brain are being mediated by different brain regions. Although self-reports are weak indications of the underlying cognitive operations, the debriefing phase of our experiments does offer some information about strategy use. In the verbal working memory experiment, both age groups universally reported using verbal rehearsal strategies, and not spatial ones. Likewise, in the spatial study, spatial strategies and not verbal ones were equally prominent for both groups. Finally, the use of a spatial strategy would be expected to lead to more errors to 
near than to far foils in the spatial-memory task (Smith \& Jonides, 1998). An analysis of errors made on this task revealed that both older and younger subjects showed this effect of distance, and it was, if anything, slightly stronger in the older group. These pieces of evidence argue against the possibility that older adults are using verbal strategies in spatial tasks, and vice versa. Instead, they favor the idea that any reorganization or compensatory recruitment that may be occurring in the older brain serves to bolster the use of the same cognitive strategies used in the younger adults.

In closing, we must consider the possibility that the age differences we observed are artifacts of the aging process, rather than indications of changes in neural function. In particular, neuroimaging studies of aging must be concerned with hemodynamic differences between older and younger brains (e.g., D'Esposito, Zarahn, Aguirre, \& Rypma, 1999). Our normalization procedures correct for age-related reductions in global blood-flow by transforming the radioactive counts obtained from each individual to a standard scale. Moreover, the key results entail age-related increases in activation which are not readily explained by age reductions in global blood flow. Of greater concern are the potentially confounding effects of localized cortical atrophy. Regions of prefrontal cortex are among those most affected by atrophic effects of aging (e.g., Raz, in press). Because the present age differences in activation are located primarily in frontal areas, they could conceivably result from region-specific atrophy in older adults, rather than from functional alterations of the working memory circuitry. While regional atrophy is likely to affect an area's function, it could also cause localized reductions in the PET signal due to reduced hemodynamic activity and partial volume effects within the atrophic regions (e.g., Labbe, Froment, Ashburner, \& Cinotti, 1996). Importantly, our results indicate that older adults show increased activation in specific frontal areas compared to younger adults. Such increases are more likely the result of age-related alterations in neural activity than regional atrophy.

\section{CONCLUSIONS}

The main result from the present set of experiments is that the anterior components of the working memory circuitry are bilaterally organized in older adults, whereas younger adults show opposite patterns of laterality for verbal and spatial working memory. There were no age differences in posterior working memory sites. Our results, therefore, suggest that the effects of normal aging on the neural substrate of working memory are selective in that the frontal components of the working memory circuitry show greater vulnerability to aging than the posterior components. The decreased anterior lateralization in older adults may be compensatory, insofar as it reflects the recruitment of addi- tional brain regions to augment task performance (cf., Reuter-Lorenz, Stanczak, \& Miller, in press). The paradoxical laterality associated with activation of DLPFC in older adults underscores the fact that aging can have highly localized effects involving executive processing regions. Understanding the functional consequences of these age differences is an important agenda item for future research.

\section{METHODS}

\section{Verbal Working Memory}

\section{Subjects}

Eight younger (21-30 years, mean age 23.3) and 16 older (65-75 years, mean age 69.9) female adults participated in this study and were paid for their time. Older adults were recruited through the University of Michigan, Institute of Gerontology, and through newspaper advertisements. Younger adults were recruited through University of Michigan subject pools and through newspaper advertisements. All participants were righthanded, free of positive neurological histories, and had normal or corrected-to-normal vision. The two age groups had similar education levels (younger: 15 years; older: 16 years).

\section{Task and Procedure}

In the verbal memory task, four upper case letters comprised the memory set on each trial. The letters were randomly chosen from the set of consonants. The letters were printed in Helvetica (24-point bold). They were positioned at the four corners of an imaginary square centered on the fixation point (see Figure 1a). The probe letter appeared in the center of the screen, replacing the fixation point, and was always lowercase. In half of the trials, the probe matched one of the letters in the memory set, and, in the other half, it did not. In the control task, the memory set consisted of only one letter, presented in each of the four corners of the imaginary square.

Each memory trial began with the onset of the fixation point in an otherwise blank field for $500 \mathrm{msec}$. Then the memory set appeared for $500 \mathrm{msec}$, followed by a 3000msec retention interval. The response period began with the appearance of the probe letter and lasted for 1500 msec. Subjects indicated whether the probe matched the identity of one of the target letters by pressing one of two keys on the mouse. As can be seen from Figure 1, each trial in the control condition lasted the same amount of time, but some of the event durations were different. The initial fixation period (or intertrial interval) was $3200 \mathrm{msec}$, followed by a 500-msec exposure of the one-item target set. After a brief 300-msec interval, the probe letter appeared for $1500 \mathrm{msec}$ during which time the subject responded. 
Subjects participated in one behavioral session to familiarize themselves with the verbal memory and control tasks. This session consisted of approximately 20 trials of practice in the memory condition, followed by 96 experimental trials. After a brief practice run in the control condition, 48 trials of experimental data were obtained. The PET session typically occurred within 7 days following this behavioral session. In the PET session, subjects participated in two scans for each of the two tasks, in a counterbalanced order. Two blocks of 24 trials were completed for each task. There were approximately 11 trials per scan.

\section{Spatial Working Memory}

\section{Subjects}

Two new groups of younger $(n=10 ; 18-25$ years, mean age $=21.2)$ and older $(n=10 ; 62-73$ years, mean age 67.4) female adults participated in the PET phase of this study. The two age groups had similar levels of education (younger: 15 years; older: 16 years). Nine additional older adults participated in only the practice phase but were excluded from the PET phase because their spatial memory accuracy performance was greater than one standard deviation below that of the younger participants. We were concerned that by including such poor performing individuals in our older sample, we would be less able to attribute group differences to the effects of age. Moreover, we wanted the age-related differences in performing the spatial task to be similar to, and certainly no greater than the age-related differences associated with verbal performance. Participants were recruited as described above.

\section{Task and Procedure}

In the memory condition, three black dots $\left(0.7^{\circ}\right.$ diameter) were presented. The dot positions were located on four imaginary circles around the fixation point, with radii subtending $3^{\circ}, 4^{\circ}, 5^{\circ}$, and $6^{\circ}$ of visual angle, respectively. For mismatch trials, the location of the probe could be either near $\left(2.4^{\circ}\right)$ or far $\left(3.2^{\circ}\right)$ from one of the target locations. In the control condition, three black dots also appeared, however, two dots remained stationary for an entire block of trials. The stationary locations were either above and below fixation, or to the left and right of fixation. The single dot that varied in location could be in any of the positions as described above for the memory trials, except those occupied by the stationary dots. The miss trials could be near or far as described above. For both memory and control blocks, half blocks contained matching probes, and the other half contained mismatching probes, equally divided between near and far misses.

The trial events were analogous to those in the verbal task (see Figure 1b). For the memory condition, a 500msec fixation period was followed by a 500-msec ex- posure of the spatial memory set. After a 3000-msec retention interval, the probe circle appeared for 1500 msec, during which time the subject pressed a mouse key to indicate whether or not the probe location matched any of the target locations. In the control task, the fixation period/intertrial interval was $3300 \mathrm{msec}$, the target display appeared for $500 \mathrm{msec}$, followed by a 200msec delay and the appearance of the probe for 1500 msec. Data from eight scans of each of the two spatial tasks was obtained. There were approximately 11 trials per scan.

\section{Image Acquisition}

A Siemens ECAT EXACT-47 PET scanner (Siemens Medical Systems, Knoxville, TN) was used in 2-D acquisition mode for the verbal experiment. Subjects were positioned in the scanner, and head position was recorded and verified before each scan. A bolus of $50 \mathrm{mCi}$ of $\left[{ }^{15} \mathrm{O}\right]$ water was delivered intravenously over a 10-sec interval. The spatial-experiment scans were acquired in 3-D mode. A $10-\mathrm{mCi}$ bolus of $\left[{ }^{15} \mathrm{O}\right]$ water was delivered over a 10-sec interval. Data were acquired for $60 \mathrm{sec}$. For 2-D studies, data acquisition was initiated when the truecoincidence event rate exceeded the random-coincidence event rate. For 3-D scans, acquisition was initiated when true coincidences reached one-half of randoms. Attenuation correction was calculated from measured transmission scans, and no correction for scattered events was performed. Data were reconstructed using filtered back projection yielding images sets with 47 contiguous slices, spaced $3.375 \mathrm{~mm}$ apart, with a resolution of $\sim 10 \mathrm{~mm}$ full-width at half-maximum (FWHM). Scans were separated by 10- (2-D) or 8-min intervals (3D) to allow for radioactive decay to near background levels.

\section{Image Analysis}

A detailed description of the image analysis protocol is given elsewhere (Jonides et al., 1997). In brief, it consisted of the following steps: (a) intrasubject registration corrected for motion between scans for each subject (Minoshima, Koeppe, Fessler, \& Kuhl, 1993a); (b) each subject's image sets were then normalized and reoriented (Minoshima, Berger, Lee, \& Mintun, 1992, 1993), and then nonlinearly warped (Minoshima, Koeppe, Frey, \& Kuhl, 1994) into a stereotactic coordinate system (Talairach \& Tournoux, 1988); (c) statistical maps were produced using in-house software (Minoshima et al., 1993a) based on the method of Friston, Frith, Liddle, \& Frackowiak (1991), except that across-subject standard deviations of the voxels averaged across the brain were used to create a pooled estimate of variance as described by Worsley, Evans, Marrett, \& Neelin (1992). A 4-pixel FWHM Gaussian filter was applied to each subject's scans prior to statistical analysis. Significance 
thresholds were calculated as derived by Friston et al. (1991).

\section{Acknowledgments}

This research was supported in part by a grant from the Office of Naval Research and in part by grants from the National Institute on Aging (NIA AG8808, AG13027), all to the University of Michigan. The research assistance of Anna Cianciolo, Elisa Rosier, Michael Kia, Edward Awh, Leon Gmeindl, and David Badre is gratefully acknowledged. The authors thank Timothy Salthouse for his comments on earlier versions of this paper.

Reprint requests should be sent to Patricia A. Reuter-Lorenz, Department of Psychology, University of Michigan, 525 E. University Ave., Ann Arbor, MI 48109-1109, or via e-mail: parl@umich.edu.

\section{Note}

1. Some male participants were also run in the verbal working memory experiment. As a group, the older males performed more poorly on the task than the older females, whose performance more closely matched the younger group. Therefore, in the verbal study, we opted to focus on the female data set to minimize confounding the effects of age and performance. Accordingly, in the spatial study, only females participated. We note, however, that in the verbal study older males, like older females, activated anterior sites in both hemispheres.

\section{REFERENCES}

Awh, E., \& Jonides, J. (1997). Spatial-selective attention and spatial-working memory. In R. Parasuraman (Ed.), The attentive brain (pp. 353-380). Cambridge, MA: MIT Press.

Awh, E., Jonides, J., Smith, E. E., Schumacher, E. H., Koeppe, R. A., \& Katz, S. (1996). Dissociation of storage and rehearsal in verbal-working memory: Evidence from positron emission tomography. Psychological Science, 7, 25-31.

Baddeley, A. (1986). Working memory. Oxford, England: Clarendon Press.

Buckner, R. L., Corbetta, M., Schatz, J., Raichle, M. E., \& Petersen, S.E. (1996). Preserved speech abilities and compensation following prefrontal damage. Proceedings of the National Academy of Science, 93, 1249-1253.

Cabeza, R., Grady, C. L., Nyberg, L., McIntosh, A. R., Tulving, E., Kapur, S., Jennings, J. M., Houle, S., \& Craik, F. I. M. (1997). Age-related differences in neural activity during memory encoding and retrieval: A positron emission tomography study. Journal of Neuroscience, 17, 391-400.

Carpenter, P. A., \& Just, M. A. (1989). The role of working memory in language comprehension. In D. Klahr \& K. Kotovsky (Eds.), Complex information processing: The impact of Herbert A. Simon (pp. 31-68). Hillsdale, NJ: Erlbaum.

Chao, L. L., \& Knight, R. T. (1997). Prefrontal deficit in attention and inhibitory control with aging. Cerebral Cortex, 7 , 63-69.

Courtney, S., Ungerleider, L. G., Keil, \& Haxby, J. V. (1996). Object, and spatial, visual-working memory activate separate neural systems in human cortex. Cerebral Cortex, 6, 39-49.

Courtney, S., Petit, L., Maisog, J., Ungerleider, L. G., \& Haxby, J. (1998). An area specialized for spatial working memory in the human frontal cortex. Science, 279, 1347-1349.

D’Esposito, M., Aguirre, G.K., Zarahn, E., Ballard, D., Shin, R. K., \& Lease, J. (1998). Functional MRI studies of spatial and nonspatial working memory. Cognitive Brain Research, 7 , $1-13$.
D’Esposito, M., Zarahn, E., Aguirre, G., \& Rypma, B (1999). The effect of normal aging on the coupling of neural activity to the bold hemodynamic response. Neuroimage, 10, 6-14.

Engelien, A., Silsberg, D., Stern, E., Huber, W., Doring, W., Frith, C., \& Frackowiak, R.S.J. (1995). The functional anatomy of recovery from auditory agnosia. Brain, 118, 13951409.

Fiez, J. A., Raife, E. A., Balota, D. A., Schwarz, J. P., Raichle, M. E., \& Petersen, S. E. (1996). A positron emission tomography study of the short-term maintenance of verbal information. Journal of Neuroscience, 16, 808-822.

Friston, K. J., Frith, C. D., Liddle, P. F., \& Frackowiak, R. S. J. (1991). Comparing functional (PET) images: The assessment of significant change. Journal of Cerebral Blood Flow Metabolism, 11, 690-699.

Goldberg, T. E., Berman, K. F., Randolph, C., Gold, J. M., \& Weinberger, D. R. (1996). Isolating the mnemonic component in spatial delayed response: A controlled PET 150-labeled water regional-cerebral blood flow study in normal humans. Neuroimage, 3, 69-78.

Goldman-Rakic, P. S. (1992). Working memory and the mind. Scientific American, 267, 110-117.

Grady C. L., Haxby, J. V., Horwitz, B., Schapiro, M. B., Rapoport, S. I., Ungerleider, L. G., Mishkin, M., Carson, R. E., \& Herscovitch, P. (1992). Dissociation of object and spatial vision in human extrastriate cortex: Age-related changes in activation of regional, cerebral blood flow measured with $\left[{ }^{15} \mathrm{O}\right]$ water and positron emission tomography. Journal of Cognitive Neuroscience, 4, 23-34.

Grady, C. L., Maisog, J. M., Horwitz, B., Ungerleider, L. G., Mentis, M. J., Salerno, J. A., Pietrini, P., Wagner, E., \& Haxby, J. V. (1994). Age-related changes in cortical bloodflow activation during visual processing of faces and location. Journal of Neuroscience, 14, 1450-1462.

Grady, C. L., McIntosh, A. R., Horwitz, B., Maisog, J. Ma., Ungerleider, L. G., Mentis, M. J., Pietrini, P., Schapiro, M. B., \& Haxby, J. V. (1995). Age-related reductions in human recognition memory due to impaired encoding. Science, 269, 218-221.

Grady, C. L., McIntosh, A. R., Bookstein, F., Horwitz, B., Rapoport, S. I., \& Haxby, J. V. (1998). Age-related changes in regional cerebral blood flow during working memory for faces. Neuroimage, 8, 409-425.

Grasby, P. M., Frith, C. D., Friston, K. J., Bench, C., Frackowiak, R. S. J., \& Dolan, R. J. (1993). Functional mapping of brain areas implicated in auditory-verbal memory function. Brain, 116, 1-20.

Ivry , R. B., \& Robertson, L. C. (1998). The two sides to perception. Cambridge MA: MIT Press.

Jonides, J. (1995). Working memory and thinking. In D. Osherson \& E. E. Smith (Eds.), An invitation to cognitive science. Cambridge, MA: MIT Press.

Jonides, J., Smith, E. E, Koeppe, R. A., Awh, E., Minoshima, S., \& Mintun, M. A. (1993). Spatial working memory in humans as revealed by PET. Nature, 363, 623-625.

Jonides, J., Reuter-Lorenz, P. A., Smith, E. E., Awh, E., Barnes, L. L., Drain, H. M., Glass, J., Lauber, E., \& Schumacher, E. (1996). Verbal and spatial working memory. In D. Medin (Ed.), Psychology of Learning and Motivation, 34, 43-88.

Jonides, J., Schumacher, E. H., Koeppe, R. A., Smith, E. E., Lauber, E., \& Awh, E. (1997). Verbal working memory load affects regional brain activation as measured by PET. Journal of Cognitive Neuroscience, 9, 462-475.

Jonides, J., Schumacher, E. H., Smith, E. E., Koeppe, R. A., Awh, E., Reuter-Lorenz, P. A., Marshuetz, C., \& Willis, C. R. (1998). The role of parietal cortex in verbal-working memory. The Journal of Neuroscience, 18, 5026-5034.

Jonides, J., Marshuetz, C., Smith, E. E., Reuter-Lorenz, P. A., 
Koeppe, R., \& Hartley, A. (in press). Changes in inhibitory processing with age revealed by brain activation. Journal of Cognitive Neuroscience.

Kinsbourne, M. (1971). The minor cerebral hemisphere as a source of aphasic speech. Archives of Neurology, 25, 302-306.

Labbe, C., Froment, J. C., Kennedy, A., Ashburner, J., \& Cinotti, L. (1996). Positron emission tomography metabolic data corrected for cortical atrophy using magnetic resonance imaging. Alzheimer's Disease and Associated Disorders, 10, 141-170.

Madden, D. J., et al. (1999). Adult age differences in functional neuroanatomy of verbal recognition memory. Human Brain Mapping, 7, 115-135.

Minoshima, S., Berger, K. L., Lee, K. S., Mintun, M. A. (1992). An automated method for rotation correction and centering of three-dimensional functional brain images. Journal of $\mathrm{Nu}$ clear Medicine, 33, 1579-1585.

Minoshima, S., Koeppe, R. A., Fessler, J. A., \& Kuhl, D. E. (1993). In K. Uemura, N. A. Lassen, T. Jones, \& I. Kanno (Eds.), Quantification of brain function-Tracer kinetics and image analysis in brain PET (pp. 409-418). Tokyo: International Congress Series 1030, Excepta Medica.

Minoshima, et al. (1993). Automated detection of the intercommissural line for stereotactic localization of functional brain images. Journal of Nuclear Medicine, 34, 322-329.

Minoshima, S., Koeppe, R. A., Frey, K. A., \& Kuhl, D. E. (1994). Anatomical standardization: Linear scaling and nonlinear warping of functional brain images. Journal of Nuclear Medicine, 35, 1528-1537.

Moscovitch, M., \& Winocur, G. (1995). Frontal lobes, memory and aging: Structure and functions of the frontal lobes. Annals of the New York Academy of Sciences, 769, 115-150.

Owen, A., Evans, A. C., \& Petrides, M. (1996). Evidence for a two-stage model of spatial working memory processing within the lateral frontal cortex: A positron emission tomography study. Cerebral Cortex, 6, 31-38.

Paulesu, E., Frith, C. D., \& Frackowiak, R. S. J. (1993). The neural correlates of the verbal component of working memory. Nature, 362, 342-344.

Petrides, M., Alivisatos, B., Meyer, E., \& Evans, A. C. (1993). Functional activation of the human frontal cortex during the performance of verbal working memory tasks. Proceedings of the National Academy of Science, 90, 878-882.

Raz, N. (in press). Aging of the brain and its impact on cognitive performance: Integration of structural and functional findings. In F. I. M. Craik \& T. A. Salthouse (Eds.), Handbook of aging and cognition-II. Mahwah, NJ: Erlbaum.
Reuter-Lorenz, P. A., Stanczak, L., \& Miller, A. (in press). Neural recruitment and cognitive aging: Two hemispheres are better than one, especially as you age. Psychological Science.

Rypma, B., Prabhakaran, V., Desmond, J. E., Glover, G. H., \& Gabrieli, J. D. (1999). Load-dependent roles of frontal brain regions in the maintenance of working memory. NeuroImage, 9, 216-226.

Salmon, E., Van der Linden, M., Collett, F., Delfiore, G., Maquet, P., Degueldre, C., Luxen, A., \& Franck, G. (1996). Regional brain activation during working-memory tasks. Brain, 119, 1617-1625.

Salthouse, T. (1991). Theoretical perspectives in cognitive aging. Hillsdale, NJ: Erlbaum.

Salthouse, T. (1992). Mechanisms of age-cognition relations in adulthood. Hillsdale, NJ: Erlbaum.

Schumacher, E. H., Lauber, E., Awh, E., Jonides, J., Smith, E. E., \& Koeppe, R. A. (1996). PET evidence for an amodal verbal working-memory system. Neuroimage, 3, 79-88.

Smith, E. E., \& Jonides, J. (1998). Neuroimaging analyses of human working memory. Proceedings of the National Academy of Science, 95, 12061-12068.

Smith, E. E., Jonides, J., \& Koeppe, R. A. (1996). Dissociating verbal- and spatial-working memory using PET. Cerebral Cortex, 6, 11-20.

Smith, E. E., Jonides, J., Koeppe, R. A., Awh, E., Schumacher, E. H., \& Minoshima, S. (1995). Spatial-versus-object working memory: PET investigations. Journal of Cognitive Neuroscience, 7, 337-356.

Talairach, J., \& Tournoux, P. (1988). Co-planar stereotaxic atlas of the buman brain. Stuttgart, NY: Thieme.

Tulving, E., Kapur, S., Craik, F. I. M., Moscovitch, M., \& Houle, S. (1994). Hemispheric encoding/retrieval asymmetry in episodic memory: Positron emission tomography findings. Proceedings of the National Academy of Sciences, USA, 91, 2012-2015.

Vallar, G., \& Shallice, T. (1990). Neuropsychological impairments of short term memory. Cambridge: Cambridge University Press.

Weiller, C., Insensee, C., Rijntjes, M., Huber, W., Muller, S., Bier, D., et al. (1995). Recovery from Wernicke's aphasia: A positron emission tomography study. Annals of Neurology, 37, $723-732$.

West, R. L. (1996). An application of prefrontal cortex theory to cognitive aging. Psychological Bulletin, 120, 272-292.

Worsley, K. J., Evans, A. C., Marrett, S., \& Neelin, P. (1992). A three-dimensional statistical analysis for rCBF activation studies in human brain. Journal of Cerebral Blood Flow Metabolism, 12, 900-918. 